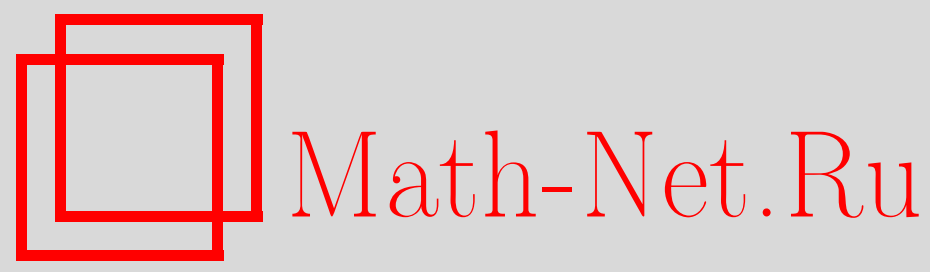

Г. А. Свиридюк, В. О. Казак, Фазовое пространство начально-краевой задачи для уравнения Хоффа, Матем. заметки, 2002, том 71, выпуск 2, 292-297

DOI: https://doi.org/10.4213/mzm347

Использование Общероссийского математического портала Math-Net.Ru подразумевает, что вы прочитали и согласны с пользовательским соглашением http://www . mathnet.ru/rus/agreement

Параметры загрузки:

IP : 107.22 .136 .117

26 апреля 2023 г., $14: 10: 27$

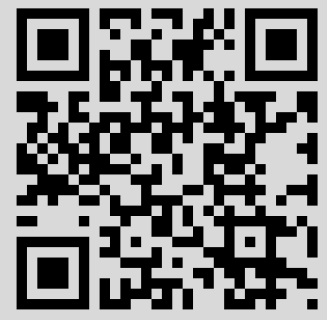




\section{ФАЗОВОЕ ПРОСТРАНСТВО НАЧАЛЬНО-КРАЕВОЙ ЗАДАЧИ ДЛЯ УРАВНЕНИЯ ХОФФА}

\section{Г. А. Свиридюк, В. О. Казак}

Уравнение Хоффа $(\lambda+\Delta) u_{t}=-\alpha u-\beta u^{3}$ описьвает динамику вьпучивания двутавровой балки. Показано, что фазовым пространством уравнения Хоффа является простое банахово $C^{\infty}$-многообразие, моделируемое подпространством, дополнительньпм к ядру $\operatorname{ker}(\lambda+\Delta)$.

Библиография: 9 названий.

Пусть $\Omega \subset \mathbb{R}^{n}$ - ограниченная область с границей $\partial \Omega$ класса $C^{\infty}$. Уравнение Хоффа

$$
(\lambda+\Delta) u_{t}=-\alpha u-\beta u^{3}
$$

в случае $n=1$ моделирует динамику выпучивания двутавровой балки [1]. Функция $u=u(x, t), x \in \Omega, t \in \mathbb{R}$, имеет физический смысл отклонения балки от положения равновесия, параметры $\lambda, \alpha, \beta \in \mathbb{R}_{+}$характеризуют нагрузку $(\lambda)$ и свойства материала $(\alpha, \beta)$. Начально-краевая задача

$$
u(x, 0)=u_{0}(x), \quad x \in \Omega, \quad u(x, t)=0, \quad(x, t) \in \partial \Omega \times \mathbb{R},
$$

для уравнения (0.1) впервые исследовалась в [2]. Здесь же была отмечена принципиальная неразрешимость задачи (0.1), (0.2) при произвольных начальных условиях. Изучение множества допустимых начальньх значений, понимаемого как фазовое пространство задачи $(0.1),(0.2)$, начато в [3], [4]. Однако в обоих случаях получены лишь частные результаты.

Статья содержит полное описание фазового пространства задачи (0.1), (0.2). Методы исследования аналогичны [4], [5]. В первой части приводятся достаточные условия разрешимости задачи Коши для полулинейного уравнения соболевского типа. Все результаты этой части вытекают из [6], [7] и поэтому снабжены лишь набросками доказательств. Во второй части абстрактные результаты прилагаются к задаче (0.1), (0.2). Отметим еще, что все рассмотрения проводятся в вещественных банаховых пространствах, однако при рассмотрении “спектральных" вопросов вводится их естественная комлексификация. Кроме того, все контуры ориентированы “движением против часовой стрелки", а символами $\mathbb{I}$ и $\mathbb{O}$ обозначены соответственно единичньй и нулевой операторы, области определения которых $(\operatorname{dom} \mathbb{I}$ и $\operatorname{dom}(\mathbb{O})$ ясны из контекста.

Работаподдержана грантами Российского фонда фундаментальных исследований.o 97-01-00444 и Минобразования РФ по направлению “Математика" № 27. 
1. Задача Коши. Пусть $\mathscr{U}$ и $\mathscr{F}$ - банаховы пространства, оператор $L \in \mathrm{Cl}(\mathscr{U}, \mathscr{F})$ (т.е. линеен, замкнут и плотно определен), оператор $M \in \mathscr{L}(\mathscr{U}, \mathscr{F})$ (т.е. линеен и непрерьвен). Следуя [6], назовем оператор $M(L, \sigma)$-ограниченным, если существует число $a \in R_{+}$такое, что для всех $\mu \in \mathbb{C}$ таких, что $|\mu|>a$, вьполняется условие $(\mu L-M)^{-1} \in \mathscr{L}(\mathscr{F}, \mathscr{U})$.

Лемма 1.1. Пусть оператор $M(L, \sigma)$-ограничен. Тогда $\mathscr{U}=\mathscr{U}^{0} \oplus \mathscr{U}^{1} u$ $\mathscr{F}=\mathscr{F}^{0} \oplus \mathscr{F}^{1}$, əде $\mathscr{U}^{0} \supset \operatorname{ker} L$, а $\mathscr{F}^{1} \subset \operatorname{im} L$. Искомые расшепления задаются проекторами

$$
P=\frac{1}{2 \pi i} \int_{\Gamma}(\mu L-M)^{-1} L d \mu, \quad Q=\frac{1}{2 \pi i} \int_{\Gamma} L(\mu L-M)^{-1} d \mu
$$

соответственно, а контур $\Gamma=\{\mu \in \mathbb{C}:|\mu|=r>a\}$.

Обозначим через $L_{k}\left(M_{k}\right)$ сужение оператора $L(M)$ на множество $\operatorname{dom} L \cap \mathcal{U}^{k}\left(\mathscr{U}^{k}\right)$, $k=0,1$.

Лемма 1.2. Пусть оператор $M(L, \sigma)$-ограничен. Тогда $L_{k} \in \mathrm{Cl}\left(\mathscr{U}^{k} ; \mathscr{F}^{k}\right)$, $M^{k} \in \mathscr{L}\left(\mathscr{U}^{k} ; \mathscr{F}^{k}\right), k=0,1$, причем существуют операторы $L_{1}^{-1} \in \mathscr{L}\left(\mathscr{F}^{1} ; \mathscr{U}^{1}\right)$ u $M_{0}^{-1} \in \mathscr{L}\left(\mathscr{F}^{0} ; \mathscr{U}^{0}\right)$.

Пусть оператор $F \in C^{k}(\mathscr{U} ; \mathscr{F}), k \in \mathbb{N} \cup\{\infty\}$. Рассмотрим задачу Коши

$$
u(0)=u_{0}
$$

для полулинейного уравнения соболевского типа

$$
L \dot{u}=M u+F(u)
$$

Пусть оператор $M(L, \sigma)$-ограничен, тогда в силу лемм 1.1 и 1.2 задача расшепляется на две задачи

$$
\begin{aligned}
G \dot{u}^{0} & =u^{0}+M_{0}^{-1} F_{0}(u), & & u^{0}=u_{0}^{0} \\
\dot{u}^{1} & =S u^{1}+L_{1}^{-1} F_{1}(u), & & u^{1}(0)=u_{0}^{1}
\end{aligned}
$$

где операторы $G=M_{0}^{-1} L_{0} \in \mathscr{L}\left(\mathscr{U}^{0}\right), S=L_{1}^{-1} M_{1} \in \mathscr{L}\left(\mathscr{U}^{1}\right), F_{0}=(\mathbb{I}-Q) F, F_{1}=Q F$, а $u^{0}=(\mathbb{I}-P) u, u_{1}=P u$.

Оператор $G$ нильпотентен степени $p \in\{0\} \cup \mathbb{N}$, тогда формально дифференцируя уравнения по $t$ и умножая слева на $G$, получим

$$
0=u^{0}+M_{0}^{-1} \sum_{q=0}^{p} H^{q} \frac{d^{q}}{d t^{q}} F_{0}(u)
$$

где оператор $H=L_{0} M_{0}^{-1} \in \mathscr{L}\left(\mathscr{F}^{0}\right)$. 
ЗАМЕчАнИЕ 1.1. Условие (1.5) вьполняется точно тогда, когда оператор $H$ нильпотентен степени $p$.

Пусть существует окрестность $O \in \mathscr{U}$ точки $u_{0} \in \mathscr{U}$ такая, что

$$
\forall u \in O \quad H F_{0 u}^{\prime} \equiv \mathbb{O}
$$

где $F_{0 u}^{\prime} \in \mathscr{L}\left(\mathscr{U} ; \mathscr{F}^{0}\right)$ - производная $\Phi$ реше оператора $F_{0} \in C^{k}\left(\mathscr{U} ; \mathscr{F}^{0}\right)$ в точке $u$. Тогда уравнение (1.5) принимает более простой вид

$$
0=u^{0}+M_{0}^{-1} F_{0}(u)
$$

Пусть $u_{0}$ - решение уравнения (1.7), причем существует оператор

$$
\left(M_{0}+F_{0 u}^{\prime}(\mathbb{I}-P)\right)^{-1} \in \mathscr{L}\left(\mathscr{F}^{0} ; \mathscr{U}^{0}\right) .
$$

Тогда в силу теоремы о неявной функции существуют окрестности $O^{0} \subset U^{0}$ и $O^{1} \subset U^{1}$ точек $u_{0}^{0}$ и $u_{0}^{1}$ соответственно и отображение $\delta \in C^{k}\left(O^{1} ; O^{0}\right)$ такие, что $u^{0}=\delta\left(u^{1}\right)$ есть решение уравнения (1.7) при любом $u^{1} \in O^{1}$, причем $u_{0}^{0}=\delta\left(u_{0}^{1}\right)$.

ЗАмЕЧАнИЕ 1.2. Если $u_{0}$ - решение уравнения (1.7), то, не теряя общности, можно положить $O=O^{0} \oplus O^{1}$.

В окрестности $O$ уравнение (1.4) приобретет вид

$$
\dot{u}^{1}=S u^{1}+L_{1}^{-1} F_{1}(\mathbb{I}+\delta)\left(u^{1}\right) .
$$

Пусть оператор $F \in C^{\infty}(\mathscr{U} ; \mathscr{F})$, тогда оператор $\delta \in C^{\infty}\left(O^{1} ; O^{0}\right)$ и в силу классической теоремы Коши [7] для некоторого $T \in \mathbb{R}_{+}$сушествует единственное решение $u^{1} \in C^{\infty}\left((-T, T) ; O^{1}\right)$ задачи (1.4). Итак, доказана

Tеорема 1.1. Пусть оператор $M(L, \sigma)$-ограничен, а оператор $F \in C^{\infty}(\mathscr{U} ; \mathscr{F})$. Пусть $u_{0} \in \mathscr{M}$, әде $\mathscr{M}=\{u \in \mathscr{U}:(\mathbb{I}-Q)(M u+F(u))=0\}$, и выполнены условия (1.5), (1.7) и (1.8). Тогда для некоторого $T=T\left(u_{0}\right)>0$ существует единственное решение $u \in C^{\infty}((-T, T) ; \mathscr{M})$ задачи (1.1), (1.2).

ДокАЗАтЕЛьство. Действительно, включение $u_{0} \in \mathscr{M}$ означает, что $u_{0}$ - решение уравнения (1.7). Поэтому искомое решение $u=u(t)$ имеет вид $u=(\mathbb{I}+\delta)\left(u^{1}\right)$. Отсюда $u(t) \in \mathscr{M}$ при любом $t \in(-T, T)$.

ЗАмечание 1.3. Следуя [3], [4], множество $\mathscr{P} \subset U$ назовем фазовылм пространством уравнения (1.2), если для любого $u_{0} \in \mathscr{P}$ существует единственное решение $u \in C^{\infty}((-T, T) ; \mathscr{P})$ задачи $(1.1),(1.2)$ при некотором $T=T\left(u_{0}\right)$. В условиях теоремы 1.1 множество $\mathscr{M} \cap O$ - фазовое пространство уравнения (1.1), являюшееся банаховым $C^{\infty}$-многообразием, моделируемьм пространством $\mathscr{U}^{1}$. 
2. Фазовое пространство. Чтобы редуцировать задачу $(0.1),(0.2)$ к задаче (1.1), (1.2), положим $\mathscr{U}=L_{4}, \mathscr{F}=W_{2}^{-1}$ (все пространства определены на области $\Omega$ ). Операторы $L, M, F$ определим формулами

$$
\begin{gathered}
\langle L u, v\rangle=\int_{\Omega}\left(\lambda u v-u_{x_{k}} v_{x_{k}}\right) d x \quad \forall u, v \in \stackrel{\circ}{W}_{2}^{1} \\
\langle M u, v\rangle=-\alpha \int_{\Omega} u v d x, \quad\langle F(u), v\rangle=-\beta \int_{\Omega} u^{3} v d x \quad \forall u, v \in L_{4},
\end{gathered}
$$

где $\langle\cdot, \cdot\rangle$ - скалярное произведение в $L_{2}$. Вложение $\stackrel{\circ}{W}_{2}^{1} \hookrightarrow L_{4}$ плотно и непрерьвно при $n \leqslant 4$, поэтому оператор $L \in \mathrm{Cl}(\mathscr{U} ; \mathscr{F})$. В [3], [4] показано, что в силу вложения $\left(L_{4}\right)^{*} \cong L_{4 / 3} \hookrightarrow W_{2}^{-1}$ оператор $F \in C^{\infty}(\mathscr{U} ; \mathscr{F})$.

По построению оператор $L$ фредгольмов (т.е. ind $L=0$ ). Кроме того, спектр $\sigma(L)$ оператора $L$ вешественен, дискретен, конечнократен и сгущается только к точке $-\infty$. Пусть точка $0 \notin \sigma(L)$, тогда в силу [8] проекторы $P=Q=\mathbb{O}$. Поэтому все условия теоремы 1.1 очевидным образом вьполнены и, значит, справедлива

Tеорема 2.1. Пусть $0 \notin \sigma(L) u n \leqslant 4$. Тогда для любого $u_{0} \in L_{4} u$ некоторого $T=T\left(u_{0}\right)$ существует единственное решение $u \in C^{\infty}\left((-T, T) ; L_{4}\right)$ задачи (0.1), (0.2).

ЗАмЕЧАнИЕ 2.1. Как нетрудно заметить, в условиях теоремы 2.1 фазовым пространством уравнения Хоффа является все пространство $L_{4}$.

Теперь, пусть $0 \in \sigma(L)$. В ядре $\operatorname{ker} L$ выберем ортонормальньй (в смысле $L_{2}$ ) базис $\left\{\varphi_{1}, \varphi_{2}, \ldots, \varphi_{m}\right\}$. В силу [8] достаточньм условием $(L, \sigma)$-ограниченности оператора является отсутствие $M$-присоединенных векторов. В нашем случае поскольку $M \psi \notin$ $\operatorname{im} L$ при любом $\psi \in \operatorname{ker} L \backslash\{0\}$, то $M$-присоединенные векторы оператора $L$ отсутствуют. Далее, проекторы $P$ и $Q$ имеют вид

$$
P=Q=\mathbb{I}-\sum_{l=1}^{m}\left\langle\cdot, \varphi_{l}\right\rangle \varphi_{l}
$$

и, значит, операторы $H=G=\mathbb{O}$. Отсюда немедленно вытекает, что условия (1.5) и (1.6) тривиально выполняются. Из (2.1) и (1.8) следует, что в фазовом пространстве уравнения Хоффа лежат те точки $u \in L_{4}$, для которых

$$
\int_{\Omega}\left(\alpha+\beta u^{2}\right) u \varphi_{l} d x=0
$$

причем определитель

$$
\left|\int_{\Omega}\left(\alpha+3 \beta u^{2}\right) \varphi_{k} \varphi_{l} d x\right| \neq 0
$$

где $k, l=1,2, \ldots, m$.

Обозначим через $\mathscr{M}$ множество решений уравнений $(2.2)$ из пространства $L_{4}$ и введем в рассмотрение множество $\mathscr{L}=\left\{u \in L_{4}:\left\langle u, \varphi_{l}\right\rangle=0, l=1,2, \ldots, m\right\}$. 
Лемма 2.1. Пусть $0 \in \sigma(L)$ u $n \leqslant 4$. Тогда для любого $v \in \mathscr{L}$ существует единственный вектор $\psi \in \operatorname{ker} L$ такой, что $u=\psi+v \in \mathscr{M}$.

ДоКАЗАТЕЛЬСТво. Воспользуемся теорией степени непрерывного отображения. Для этого фиксируем точку $v \in \mathscr{L}$ и, пользуясь $(2.2)$, построим отображение

$$
f: \psi \rightarrow\left(\beta \int_{\Omega}(\psi+v)^{3} \psi d x+\alpha\|\psi\|_{2}^{2}\right) \psi .
$$

(Здесь и далее $\|\cdot\|_{k}-$ норма пространства $L_{k}$.) Как нетрудно видеть, $f \in C(\operatorname{ker} L, \operatorname{ker} L)$. Далее, из (2.4) в силу неравенства Гёльдера получим

$$
\langle f(\psi), \psi\rangle \geqslant\|\psi\|_{2}^{2}\|\psi\|_{4}\left(\beta\|\psi\|_{4}^{3}-3 \beta\|\psi\|_{4}^{2}\|v\|_{4}-3 \beta\|\psi\|_{4}\|v\|_{4}^{2}-\beta\|v\|_{4}^{3}-\alpha c^{2}\|\psi\|_{4}^{2}\right),
$$

где $c$ - константа вложения $L_{2} \hookrightarrow L_{4}$. Отсюда вытекает, что $\langle f(\psi), \psi\rangle>0$ на сфере радиуса $R>3\left(\|v\|_{4}+\|v\|_{4}^{2}+\|v\|_{4}^{3}+c^{2} \alpha(3 \beta)^{-1}\right)$.

Далее, пусть $u$ и $v$ - два решения (2.2) при фиксированном $v \in \mathscr{L}$, т.е. $u=\xi+v$ и $w=\eta+v$. Тогда

$$
0=\alpha \int_{\Omega}(\xi-\eta)^{2} d x+\beta \int_{\Omega}(\xi-\eta)^{2}\left(u^{2}+u w+w^{2}\right) d x .
$$

Поскольку $u^{2}+u w+w^{2}>0$ кроме случая $u=v=0$, то из (2.5) вытекает, что $u=w$.

Теорема 2.2. Пусть $0 \in \sigma(L) u n \leqslant 4$. Тогда при любом $u_{0} \in \mathscr{M} u$ некотором $T=T\left(u_{0}\right)>0$ существует единственное решение $u \in C^{\infty}((-T, T) ; \mathscr{M})$ задачи (0.1), (0,2).

ДокАЗАТЕЛЬСТво. Обозначим через $A(u)$ матрицу, определитель которой фигурирует в (2.3). Предположим, что $\operatorname{det} A(u)=0$ для некоторой точки $u \in \mathscr{U}$. Тогда существует вектор $\psi \in \operatorname{ker} L \backslash\{0\}$ такой, что $A(u) \psi=0$. Однако

$$
\langle A(u) \psi, \psi\rangle=\int_{\Omega}\left(\alpha+3 \beta u^{2}\right) \psi^{2} d x>0 .
$$

Поэтому теорема справедлива в силу теоремы 1.1 .

ЗАмЕчАнИЕ 2.2. В силу леммы 1.2 и теоремы 2.2 фазовьм пространством уравнения Хоффа служит простое $C^{\infty}$-многообразие, моделируемое пространством $\mathscr{L}$. (Напомним [5], что гладкое многообразие назьвается простым, если существует его атлас, содержащий единственную карту.)

ЗАмЕчАниЕ 2.3. В силу теорем 2.1 и 2.2 точка 0 является стационарным решением задачи $(0.1),(0.2)$ и в любом случае лежит в фазовом пространстве уравнения Хоффа. Это говорит об отсутствии в задаче $(0.1),(0.2)$ бифуркаций типа бифуркации Эйлера.

ЗАмечание 2.4. Рассмотрим случай $\operatorname{dim} \operatorname{ker} L=1$, т.e. $\operatorname{ker} L=\operatorname{span}\{\varphi\},\|\varphi\|_{2}=1$. Согласно лемме 2.1 для любого $v \in \mathscr{L}$ существует единственное число $s_{0} \in \mathbb{R}$ такое, что вектор $s_{0} \varphi+v \in \mathscr{M}$. Другими словами, $s_{0}-$ единственньй корень уравнения

$$
s^{3}\|\varphi\|_{4}^{4}+3 s^{2} \int_{\Omega} \varphi^{3} v d x+s\left(\int_{\Omega} \varphi^{2} v^{2} d x+\alpha \beta^{-1}\right)+\int_{\Omega} \varphi v^{3} d x=0 .
$$

Множество пар $(s, v) \in \mathbb{R} \times \mathscr{L}$, удовлетворяющих (2.6), названо в [9] 2-сборкой Уитни. Простота этого множества как гладкого банахова многообразия - удивительный факт по сравнению с результатами [9]. 


\section{СПИСОК ЦИТИРОВАННОЙ ЛИТЕРАТУРЫ}

[1] Вольмир А. С. Устойчивость деформируемых систем. М.: Наука, 1967.

[2] Сидоров Н. А., Романова О. А. О применении некоторых результатов теории ветвления при решении дифференциальных уравнений // Дифференц. уравнения. 1983. Т. 19. №9. C. $1516-1526$.

[3] Свиридюк Г. А., Сукачева Т. Г. Фазовые пространства одного класса операторных полулинейных уравнений типа Соболева // Дифференц. уравнения. 1990. Т. 26. № 2. С. 250-258.

[4] Свиридюк Г.А. Квазистационарные траектории полулинейных динамических уравнений типа Соболева // Изв. РАН. Сер. матем. 1993. Т. 57. № 3. С. 192-207.

[5] Свиридюк Г.А., Якупов М. М. Фазовое пространство начально-краевой задачи для системы Осколкова // Дифференц. уравнения. 1996. Т. 32. № 11. С. 1538-1543.

[6] Свиридюк Г. А. К общей теории полугрупп операторов // УМН. 1994. Т. 49. № 4. С. 47-74.

[7] Ленг С. Введение в теорию дифференцируемых многообразий. Волгоград: Платон, 1996.

[8] Свиридюк Г. А., Сукачева Т. Г., Дудко Л. Л. Относительная $\sigma$-ограниченность линейных операторов // Изв. вузов. Матем. 1997. № 7. С. 68-73.

[9] Бокарева Т. А., Свиридюк Г. А. Сборки Уитни фазовых пространств некоторых полулинейных уравнений типа Соболева // Матем. заметки. 1994. Т. 55. № 3. С. 3-10.

Челябинский государственный университет

Поступило

E-mail: ridyu@cgu.chel.su, kazak@math.cgu.chel.su

26.01.2000 